\title{
Unawareness of Involuntary Nature of Hemichorea-hemiballism due to Acute Cortical Stroke
}

\author{
Judith Navarro-Otano ${ }^{1}$, Juan J Mengual ${ }^{2}$, Alvaro Cervera ${ }^{3}$, Yaroslau Compta ${ }^{1}$ and Francesc Valldeoriola ${ }^{1^{*}}$ \\ ${ }^{1}$ Parkinson's Disease and Movement Disorders Unit, Neurology Service, Hospital Clinic, University of Barcelona, Spain \\ ${ }^{2}$ Neurology Service, Hospital Moises Broggi, Sant Joan Despi, Spain \\ ${ }^{3}$ Stroke Unit, Neurology Service, Hospital Clinic, University of Barcelona, Spain
}

"Corresponding author: Francesc Valldeoriola, Parkinson's Disease and Movement Disorders Unit, Neurology Service, Clinical Institute of Neuroscience, Hospital Clinic de Barcelona, Spain, E-mail: fvallde@clinic.cat

Received date: Apr 24, 2014 Accepted date: Aug 12, 2014 Published date: Aug 17, 2014

Copyright: (c) 2014 Navarro-Otano J, et al. This is an open-access article distributed under the terms of the Creative Commons Attribution License, which permits unrestricted use, distribution, and reproduction in any medium, provided the original author and source are credited.

\section{Commentary}

Hemichorea-hemiballism is an uncommon complication from acute stroke (less than 1\%) [1]. Although it is more commonly associated with basal ganglia lesions, hyperkinetic movements due to cortical infarction sparing subcortical structures are not unusual. A recent review has reported that 27 out of 2009 patients demonstrated hemichorea after a stroke and among them 6 suffered from a cortical stroke [2]. Denial of these symptoms is a rare phenomenon that has been reported in basal ganglia but not in cortical lesions [3-5], though anosognosia is well-recognised with (mostly right-sided) parietal damage [6]. We are reporting a case of denial of the involuntary nature of acute hemichorea-hemiballism in a patient with right cortical infarction, sparing the basal ganglia.

A 51-year old, righted-handed man, without any known past medical history or treatment, was admitted to our Stroke Unit in June, 2011 because of regressive paralysis of the left limbs, followed three hours later by acute hemichorea-hemiballism involving face, neck, and left arm and leg. Upon admission, the patient was fully conscious. Visual fields and extraocular movements were normal. Muscle strength was preserved in all four limbs and plantar responses were flexor. There was a light decrease in tactile and thermal sensation in the left limbs with tactile extinction upon simultaneous touch of the legs but not of the arms. Distal choreic movements, as well as ballistic proximal movements of the left arm and leg were observed. The patient tried to redirect the ballistic movements through pseudopurpose ones, denying them being involuntary, providing odd explanations for his ballistic movements. As an example, the patient claimed that his involuntary arm jerks towards the opposite shoulder were with the intent to scratch his shoulder.

A computerized tomography performed six hours after the onset of the symptoms, showed a hypodense area in the vascular field, supplied by the right middle cerebral artery. An MRI with $1.5 \mathrm{~T}$ dWI sequences was performed within the first 24 hours. It showed an acute ischemic stroke involving the right temporo-parietal cortex, the insular cortex and the operculum. The basal ganglia were totally spared (Figure 1). The first vascular ultrasound performed while movements were present, showed a TIBI 3 pattern in the right middle cerebral artery, suggestive of a distal occlusion. The doppler ultrasound and the angioMRI performed after the movements had disappeared, were normal.

A few hours after admission, there was just a hint of the initial abnormal movements, and these quickly subsided thereafter. Throughout the entire process, the patient acknowledged the movements but denied them being involuntary. He was discharged and prescribed acetylsalicylic acid 100mg/day and atorvastatin $80 \mathrm{mg} /$ day.

Our hypothesis is that the cortical right lesion, involving the temporo-parietal junction, could have been responsible for both the involuntary movement as well as the lack of awareness of the involuntary nature of the movement. The mechanistic basis for the involuntary movement might be due to either the interruption of excitatory cortical projections to basal ganglia, with secondary dysfunction of striatum [7], or sensory deafferentation and subsequent lack of cortical inhibition of the motor output [6]. In this regard, metabolic investigations such as an SPECT/PET which were not available in this case, might have highlighted hypometabolism of the striatum and/or frontal cortex (disconnection syndrome or diaschisis). On the other hand, direct involvement of highly associative parietal areas, implied in self-awareness, might have accounted for the denial of the involuntary movements in our patient. The insula, which was acutely damaged in this case as well, has also been involved in the integration of self-awareness of one's body parts' functioning [8].

The quick recovery of the involuntary movement may be due to an acute transient ischemic functional damage to the corticobasal circuit. After a complete diagnostic work-up, the stroke was classified as undetermined etiology according to TOAST classification [9].

We acknowledge that an acute EEG might have helped to rule out the possibility of partial seizures. Still, this possibility was unlikely from a clinical point of view, since the movement was not repetitive nor was it stereotyped. Myoclonus was not present at any time and consciousness was fully preserved.

A similar case with insular infarction and anosognosia of the resulting chorea was described in 1991 [5]. Basal ganglia involvement was present then also, however it was different to the limited cortical damage in our case. Furthermore, our patient did not have proper anosognosia of the abnormal movement itself (he was aware of his limbs moving), but of the involuntary nature of these movements.

In conclusion, though cortical right lesions with secondary negligence are quite common, this is the first report of unawareness of the involuntary stroke-induced abnormal movement in the absence of basal ganglia damage. 
Citation: Navarro-Otano J, Mengual JJ, Cervera A, Compta Y and Valldeoriola F (2014) Unawareness of Involuntary Nature of Hemichoreahemiballism due to Acute Cortical Stroke. J Neurol Neurophysiol 5: 217. doi:10.4172/2155-9562-5-1000217

Page 2 of 2

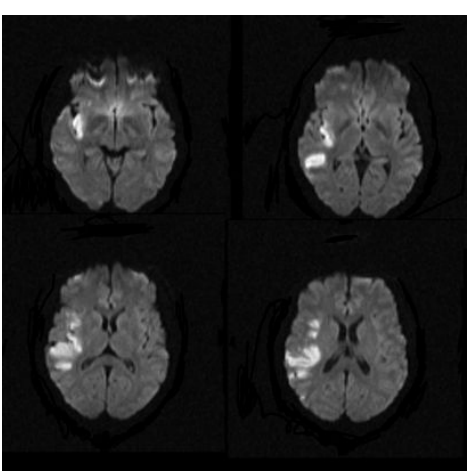

Figure 1: MR- diffusion image showing acute ischemic stroke involving right temporo-parietal cortex, insular cortex and the operculum.

\section{References}

1. Cardoso F, Seppi K, Mair KJ, Wenning GK, Poewe W (2006) Seminar on choreas. Lancet Neurol 5: 589-602.
2. Chung SJ, Im JH, Lee MC, Kim JS (2004) Hemichorea after stroke: clinical-radiological correlation. J Neurol 251: 725-729.

3. Ichikawa H, Hasegawa Y, Kawamura M, Fukui T, Sugita K (1995) A case of hemichorea presenting with inattention and denial of involuntary movement. Rinsho Shinkeigaku 35: 1230-1234.

4. Kawamura M, Takahashi N, Hirayama K (1988) Hemichorea and its denial in a case of caudate infarction diagnosed by magnetic resonance imaging. J Neurol Neurosurg Psychiatry 51: 590-591.

5. Lazzarino LG, Nicolai A (1991) Hemichorea-hemiballism and anosognosia following a contralateral infarction of the caudate nucleus and anterior limb of the internal capsule. Riv Neurol 61: 9-11.

6. Karnath HO, Baier B (2010) Right insula for our sense of limb ownership and self-awareness of actions. Brain Struct Funct 214: 411-417.

7. Young AB PJ (2002) Biochemical and functional organization of the basal ganglia. In: Jankovic J TE, ed. Parkinsonâ $€^{\mathrm{m}} \mathrm{s}$ disease and movement disorders. Philadelphia: Lippincott Williams \& Wilkins 1-10.

8. Karnath HO, Baier B, Nägele T (2005) Awareness of the functioning of one's own limbs mediated by the insular cortex? J Neurosci 25: 7134-7138.

9. Adams HP Jr, Bendixen BH, Kappelle LJ, Biller J, Love BB, et al. (1993) Classification of subtype of acute ischemic stroke. Definitions for use in a multicenter clinical trial. TOAST. Trial of Org 10172 in Acute Stroke Treatment. Stroke 24: 35-41. 